\title{
Folding of Protein Ions in the Gas Phase after
}

\section{Cation to Anion Proton Transfer Reactions (CAPTR)}

\author{
Kenneth J. Laszlo, Eleanor B. Munger, Matthew F. Bush*
}

Contribution from the:

University of Washington, Department of Chemistry, Box 351700

Seattle, WA 98195-1700

Email: mattbush@uw.edu

\section{SUPPORTING INFORMATION}

*Address correspondence to this author. 


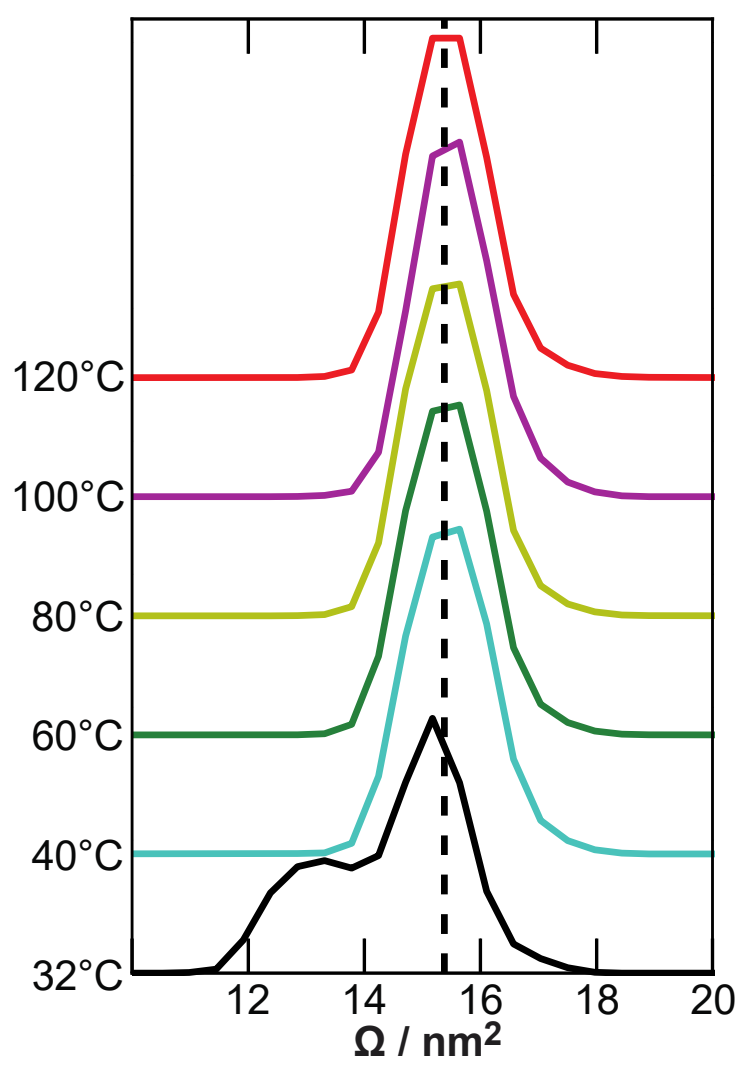

Figure S1: The $P \rightarrow 7 \Omega$ distributions in Figure 3 exhibit a monomodal arrive-time distribution corresponding to a $\Omega$ of $15.4 \mathrm{~nm}^{2}$. Previous results showed distributions spanning 9 to $17 \mathrm{~nm}^{2}$, some of which exhibited multi-modal distributions. ${ }^{1-4}$ One explanation for the single, monomodal feature is that these experiments used a source temperature of $120^{\circ} \mathrm{C}$, which may activate the proteins during ionization or in solution due to convective heat transfer. Figure S1 shows the $\Omega$ distribution of $7+$ ubiquitin as a function of source temperature. The vertical black dashed line indicates the average $\Omega$ of $P \rightarrow 7$ ion in Figure 4 . At $32^{\circ} \mathrm{C}$, the ion has a bimodal distribution from 12 to $17 \mathrm{~nm}^{2}$, and shifts to a monomodal distribution centered around $15.4 \mathrm{~nm}^{2}$ with the source held at $40^{\circ} \mathrm{C}$. This is generally consistent with previous experiments in which increasing the inlet capillary from 25 to $132{ }^{\circ} \mathrm{C}$ resulted in the formation $7+$ ubiquitin ions that exhibited a $\Omega$ distribution ${ }^{1}$ similar to that observed here. 


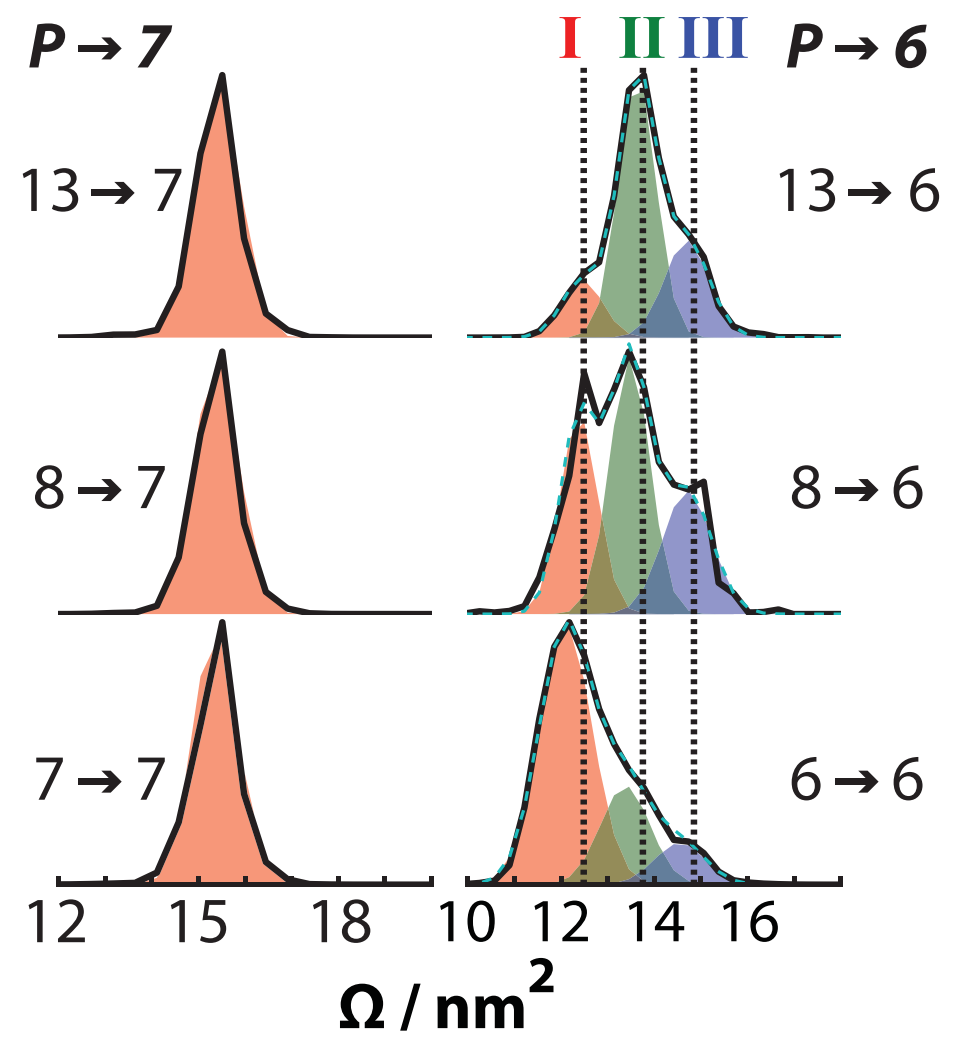

Figure S2. $\Omega$ distributions of selected $P \rightarrow 7$ and $P \rightarrow 6$ ubiquitin ions as a function of the charge state of the precursor ion $(P)$. The data for the $P \rightarrow 7$ ions is the same as that used to determine the centroid $\Omega$ reported in Figure 4. The data for the $P \rightarrow 6$ ion is the same at the reported in Figure 5A to $5 \mathrm{C}$ using a trap injection voltage of $45 \mathrm{~V}$. The $P \rightarrow 7 \Omega$ distribution are well-described using a single Gaussian function. The $\Omega$ distributions of the $P \rightarrow 6$ ions were assigned using three Gaussian functions; the sum of the three functions is shown with a dashed cyan line. The centroids for the all $P \rightarrow 7$ Gaussian functions, and the three $P \rightarrow 6$ Gaussian functions for $P=13+$ to $6+($ Figure 4$)$ were averaged and those four averages are shown using dashed black lines. 

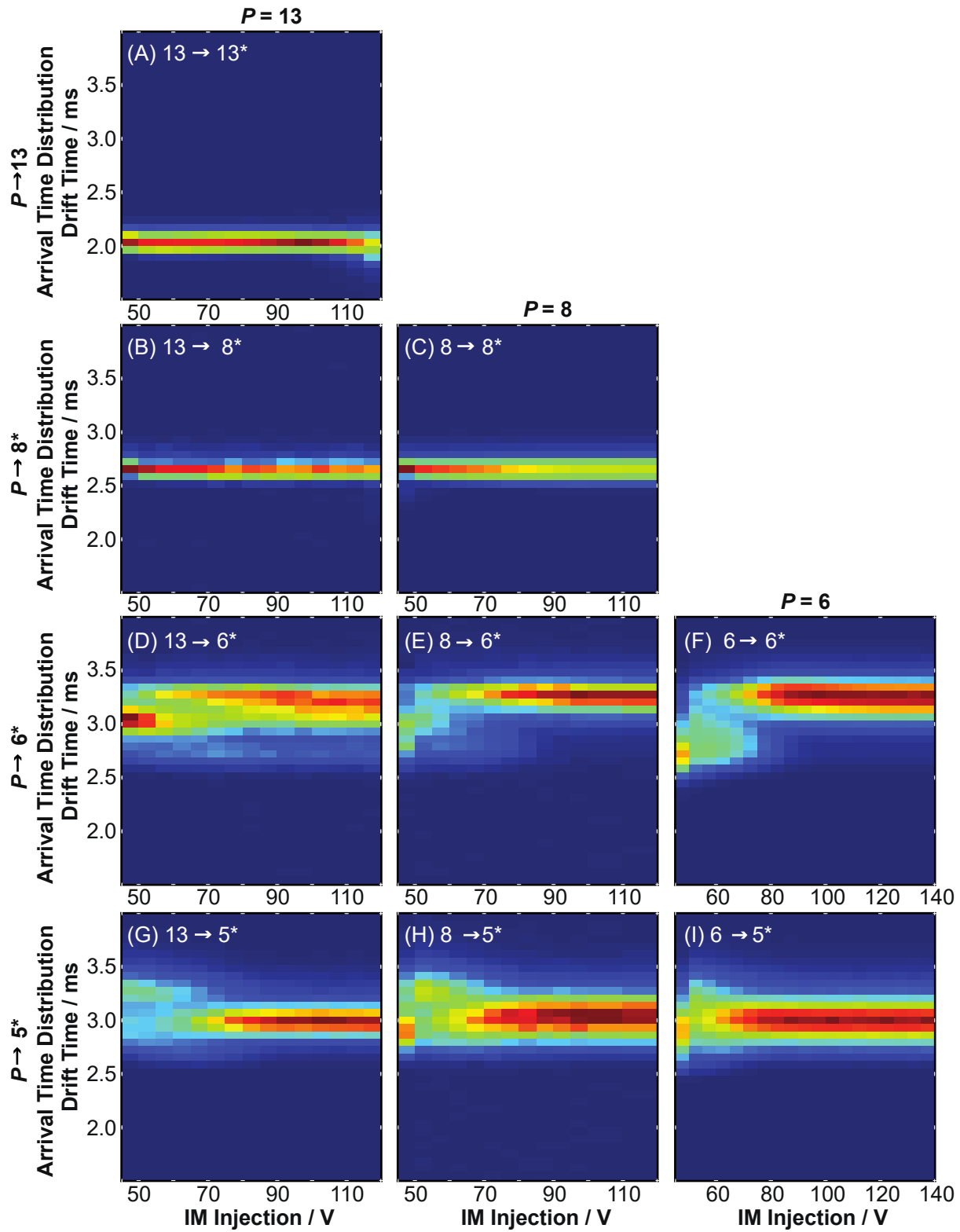

Figure S3. Arrival-time distributions for selected CAPTR product ions from precursor charge states $(P) 13+, 8+$, and $6+$ ubiquitin plotted against injection voltage into mobility cell. 

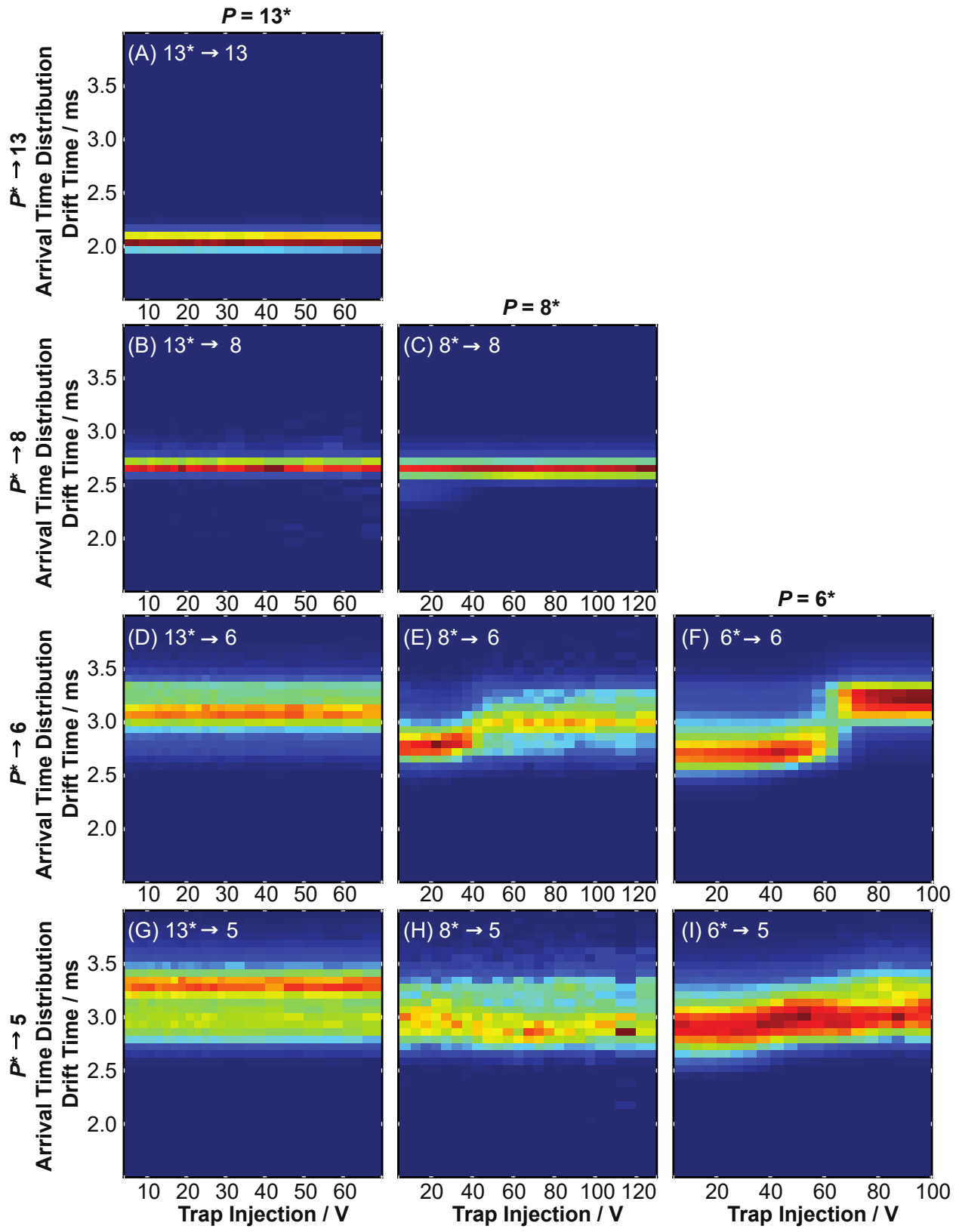

Figure S4. Arrival-time distributions of $P \rightarrow 13, P \rightarrow 8, P \rightarrow 6$, and $P \rightarrow 5$ ubiquitin product ions $(P)$ and from precursor charge states $13+, 8+$, and $6+$ when activated entering the trap cell. 
Consideration of Spontaneous Charge Loss: One challenge for interpreting these results is that ions adducted with any additional salt clusters may undergo the spontaneous loss or gain of charge following IM separation and prior to mass analysis. This is attributed to the ion ejecting either positively or negatively charged salt/solvent clusters when provided adequate collisional activation. These ions would therefore have the mobility of a $P$ ion during the IM separation, but the $m / z$ of a $(P-1)+$ or $(P+1)$ ion during mass analysis. ${ }^{5,6}$ For these experiments we can estimate how significant these pathways are by comparing the intensities of the quadrupole selected peak, $P$, and the corresponding anion ejected peak, $(P+1)$. For the data collected, the $13+$ precursor ion showed no sign of anion loss to generate a $14+$ cation. However, when the $8+$ ion was selected, there is a low-intensity signal corresponding to a 9+ cation at high collision energies. With the highest collisional activation into the trap cell, the $9+$ to $8+$ ratio was 0.0026 , indicating the loss of an anion is not a dominant pathway in these experiments. We expect the rate of anion loss to be less than the rate of cation loss, however these values are expected to be on a similar order of magnitude. 


\section{Determining Collision Cross Sections:}

Measured drift times (tD) in these experiments depend on the mobility dependent drift time ( $\left.\mathrm{tK}_{\mathrm{K}}\right)$ and the transport time of ions from the exit of the mobility cell to the time-of-flight mass analyzer $\left(\mathrm{t}_{0}\right)$ :

$$
t_{D}=t_{K}+t_{0}
$$

where the $t_{\mathrm{K}}$ is defined as:

$$
\mathrm{t}_{\mathrm{k}}=\frac{\text { mobility cell length }}{\mathrm{KE}}
$$

where $\mathrm{K}$ is the mobility of the ion and $\mathrm{E}$ is the electric field in the mobility cell

Measuring the drift time of nESI generated ubiquitin cations (identical denaturing conditions) at 10 electric fields from 104 to $354 \mathrm{~V}$ drop across the $25.2 \mathrm{~cm}$ mobility cell was used to determine $t_{0}$. $t_{0}$ has both a $\mathrm{m} / \mathrm{z}$ dependent $\left(\mathrm{t}_{\mathrm{m} / \mathrm{z}}\right)$ and independent term $\left(\mathrm{t}_{\text {ind }}\right)$, which is related to instrumental parameters. tind is defined as:

$$
\mathrm{t}_{\text {ind }}=\mathrm{t}_{0}-\mathrm{t}_{\mathrm{m} / \mathrm{z}}
$$

$t_{m / z}$ is defined as: ${ }^{7}$

$$
\mathrm{t} m / z=\frac{c \sqrt{m / z}}{1000}
$$

where $c$ is an instrument specific parameter (the 'enhanced dude cycle delay coefficient' on the Synapt G2). The average of tind from field dependent measurements was treated as a constant for CAPTR-IM experiments. For example, the average tind for charge states $13+$ through $9+$ ubiquitin was $0.04 \mathrm{~ms}$, with a standard deviation of $0.005 \mathrm{~ms}$.

CAPTR-IM experiments were performed at 1.5 Torr Helium with a $127 \mathrm{~V}$ drop across the mobility cell (unless otherwise noted). For CAPTR precursor and product ions, to was calculated by summing tind and $t_{m / z}$, using the $m / z$ value of each respective ion. For example, for ubiquitin charge states $13+$ through $3+t_{m / z}$ ranged from 0.04 to 0.08 . $t_{0}$ and measured tD were 
then used to calculate K of CAPTR product ions and residual precursors (Equation S2). Collision cross section $(\Omega)$ values were determined from the experimental K using the Mason-Schamp equation: ${ }^{8}$

$$
\Omega=\frac{3 e z}{16 \mathrm{~N}}\left(\frac{2 \pi}{\mu \mathrm{k}_{\mathrm{B}} \mathrm{T}}\right)^{1 / 2} \frac{1}{\mathrm{~K}}
$$

where $e$ is the fundamental charge, $\mathrm{N}$ is the number density of the drift gas, $\mu$ is the reduced mass of the ion and drift gas, $\mathrm{k}_{\mathrm{B}}$ is the Boltzmann constant, and $\mathrm{T}$ is the drift-gas temperature 


\section{References}

(1) Li, J.; Taraszka, J. A.; Counterman, A. E.; Clemmer, D. E. Int. J. Mass Spectrom. 1999, 185-187, $37-47$.

(2) Wyttenbach, T.; Bowers, M. T. J. Phys. Chem. B 2011, 115 (42), 12266-12275.

(3) Shi, H.; Atlasevich, N.; Merenbloom, S. I.; Clemmer, D. E. J. Am. Soc. Mass Spectrom. 2014, 25 (12), 2000-2008.

(4) Shi, H.; Clemmer, D. E. J. Phys. Chem. B 2014, 118 (13), 3498-3506.

(5) Freeke, J.; Bush, M. F.; Robinson, C. V.; Ruotolo, B. T. Chem. Phys. Lett. 2012, 524, 1-9.

(6) Sobott, F.; McCammon, M. G.; Robinson, C. V. Int. J. Mass Spectrom. 2003, 230 (2-3), 193-200.

(7) Ruotolo, B. T.; Benesch, J. L.; Sandercock, A. M.; Hyung, S. J.; Robinson, C. V. Nat Protoc 2008, 3, 1139-1152.

(8) Mason, E. A.; McDaniel, E. W. Transport Properties of Ions in Gases; Wiley: New York, 1988. 